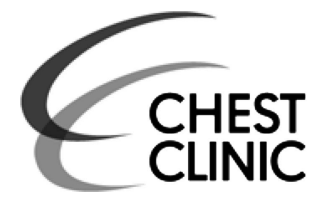

\title{
The cost of treating severe refractory asthma in the UK: an economic analysis from the British Thoracic Society Difficult Asthma Registry
}

\author{
Stephen O'Neill, ${ }^{1}$ Joan Sweeney, ${ }^{2}$ Chris C Patterson, ${ }^{3}$ Andrew Menzies-Gow, ${ }^{4}$ \\ Rob Niven, ${ }^{5}$ Adel H Mansur, ${ }^{6}$ Christine Bucknall, ${ }^{7}$ Rekha Chaudhuri, ${ }^{8}$ Neil \\ C Thomson, ${ }^{8}$ Chris E Brightling, ${ }^{9}$ Ciaran O'Neill, ${ }^{1}$ Liam G Heaney, ${ }^{2}$ on behalf of the \\ British Thoracic Society Difficult Asthma Network
}

- Additional material is published online only. To view please visit the journal online (http://dx.doi.org/10.1136/ thoraxjnl-2013-204114).

For numbered affiliations see end of article.

\section{Correspondence to}

Professor Liam Heaney, Centre for Infection and Immunity, Queen's University of Belfast, Level 8, Belfast City Hospital, Lisburn Road, Belfast, Northern Ireland BT9 7AB, UK; I.heaney@qub.ac.uk

Received 8 July 2013 Revised 28 April 2014 Accepted 22 May 2014 Published Online First 10 June 2014

\section{ABSTRACT}

Severe refractory asthma poses a substantial burden in terms of healthcare costs but relatively little is known about the factors which drive these costs. This study uses data from the British Thoracic Society Difficult Asthma Registry $(n=596)$ to estimate direct healthcare treatment costs from an National Health Service perspective and examines factors that explain variations in costs. Annual mean treatment costs among severe

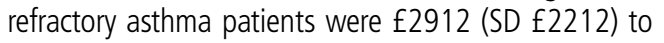
$£ 4217$ (SD £2449). Significant predictors of costs were $\mathrm{FEV}_{1} \%$ predicted, location of care, maintenance oral corticosteroid treatment and body mass index. Treating individuals with severe refractory asthma presents a substantial cost to the health service.

\section{INTRODUCTION}

The global prevalence of asthma is estimated to be 300 million $^{1}$ and approximately $18 \%$ of the UK population have doctor diagnosed asthma. ${ }^{2}$ There is a significant economic burden associated with the disease $^{3}$ and an association between disease severity and economic burden. ${ }^{4}$

A key issue in prior analyses is the definition of severe asthma. This paper focuses on patients precisely characterised using systematic evaluation protocols as having severe refractory asthma (SRA) and explores the direct annual treatment costs and drivers of cost within this population from the perspective of a publicly funded provider.

\section{METHODS}

The data analysed are patient-specific anonymised healthcare data drawn from the British Thoracic Society National Registry for dedicated UK Difficult Asthma Services in 2012. Currently, seven UK Specialist Difficult Asthma Services submit data to the Registry: Belfast City Hospital; Royal Brompton Hospital, London; Glenfield Hospital, Leicester; University Hospital of South Manchester; Birmingham Heartlands Hospital; Gartnavel Hospital, Glasgow; and Stobhill Hospital, Glasgow. After systematic multi-disciplinary assessment, patients were classified using the American Thoracic Society ${ }^{5}$ definition for refractory asthma $(n=516)$ and compared with a group with difficult to manage, but non-SRA disease $(n=80)$.
Data from the Registry capture healthcare utilisation including hospital admissions and general practitioner/Accident \& Emergency (GP/A\&E) unscheduled visits in the 12 months prior to being first seen at the difficult asthma clinics. Published references were used to monetise healthcare utilisation and each aspect of care was multiplied by this cost and aggregated to provide a total healthcare cost for the individual. As costs are estimated for a 12-month time period, no discounting is required.

All asthma-related medications were recorded at the patient's first visit to the Difficult Asthma Service. Unit costs for medication were obtained from the Prescription Cost Analyses for Northern Ireland 2011.

Where data were not captured in the Registry, estimates were made based on expert opinion, using high/low cost scenarios. Scheduled GP visits were estimated at one visit per year in the low cost scenario and in the high cost scenario were matched to unscheduled visits, where it was assumed that each unscheduled GP/A\&E visit would be followed up with a scheduled GP visit. Outpatient respiratory reviews were estimated at two visits per year and include the cost of consultant/nurse time and lung function tests. Additional detail on the low/high cost scenario is presented in the online supplementary material.

In addition to asthma treatments, the Registry records any other medication received by the patients. These were costed using a similar methodology and are listed as non-asthma medication. Asthma and non-asthma medication were identified and categorised before analyses. Full references are available in the online supplementary material.

\section{DATA ANALYSIS}

After calculating total costs for each patient, a series of regression analyses were used to examine factors that explain variations in treatment costs among SRA patients. Variables used to explain costs (chosen based on available data) were gender, age, clinical centre, best achieved $\mathrm{FEV}_{1}$, use of maintenance oral corticosteroids (OCS), body mass index (BMI) and smoking status. Analysis was carried out using Stata 10. 


\section{RESULTS}

Figure 1 displays costs for healthcare utilisation and asthma-related medication for SRA patients (see online supplementary figure S1 for non-SRA costs), a breakdown of costs (see online supplementary table A1), summary of demographic data (see online supplementary table A2), medication costs in the low/high cost scenarios (see online supplementary figures S2A (SRA patients) and S2B (non-SRA patients)) and a breakdown of medication, healthcare utilisation and associated costs (see online supplementary tables A3-A7) together with comparisons by hospital and of patient characteristics.

SRA patients had more unscheduled visits (SRA, median 4 (IQR 2-6) vs non-SRA, median 3 (IQR 0-6)) and hospital admissions (SRA, median 0 (IQR 0-1), 274 of 516 (48\%) had no admissions vs non-SRA, median 0 (IQR 0-1), 43 of 80 (54\%)), confirming that the admission rate in SRA patients in these specialist centres was relatively low at referral (see online supplementary table A2). The combined average cost of these medical services in the low (high) cost scenario was $£ 1207$ ( $£ 2077)$ for SRA patients and $£ 935$ ( $£ 1764$ ) for non-SRA patients.

The major driver of cost was medication (see online supplementary figure S2). Long acting bronchodilator/corticosteroid combination inhalers represent the greatest share of medicationrelated costs ( $£ 885-£ 1239$ for SRA patients (low/high cost scenario) and $£ 425-£ 678$ for non-SRA patients). Small numbers of patients on oxygen (five SRA) and omalizumab (11 SRA) incurred substantial costs (oxygen, individual patient cost $£ 8908$ and omalizumab, costing between $£ 3330$ and $£ 26640$ depending on omalizumab dose) and cost differences were also noted between clinical centres (see online supplementary table A7).

Regression analysis (online supplementary tables A8-A11, low cost scenario) revealed that, after adjustment for covariates, patients on maintenance OCS on average cost $43 \%$ more than those not on maintenance steroids (95\% CI $27 \%$ to 59\%). Of note also is that non-medication costs (19\% greater (95\% CI $-4 \%$ to $42 \%)$ ) and non-asthma-related medication were also higher (58\% greater (95\% CI 11\% to 104\%)) for these patients. Similar results were evident in the high cost scenario (see online supplementary tables A8-11). Patients requiring two or more rescue

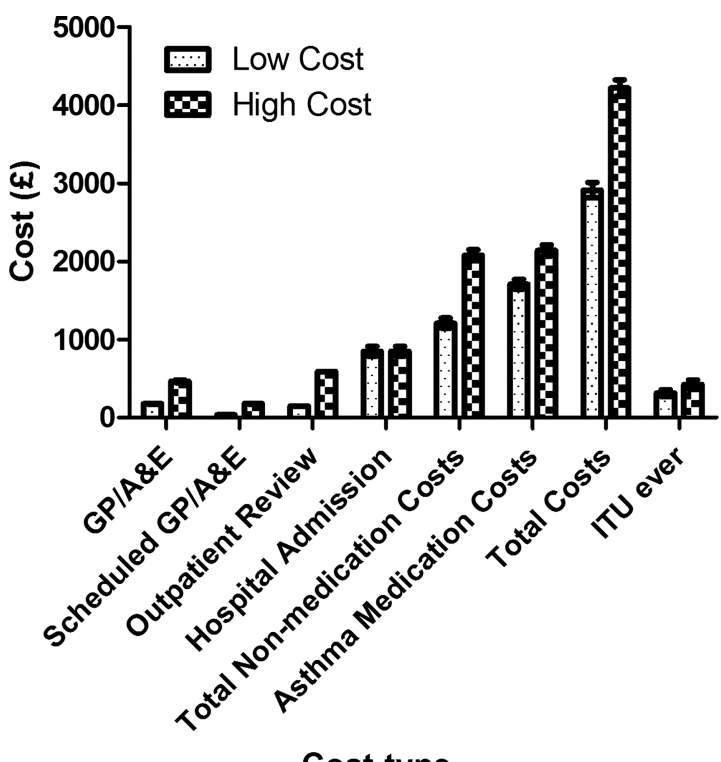

\section{Cost type}

Figure 1 Healthcare and asthma-related medication costs for severe refractory asthma. courses of steroids had $31 \%$ higher costs (95\% CI $14 \%$ to $48 \%$ ) illustrating the importance of recurrent exacerbations. For each unit increase in percentage of predicted $\mathrm{FEV}_{1}$, there was an associated $0.34 \%$ reduction in asthma-related medication costs $(95 \%$ $\mathrm{CI}-0.55 \%$ to $-0.12 \%)$. In the high cost scenario, total costs for severely obese SRA patients (BMI $>40 \mathrm{~kg} / \mathrm{m}^{2}$ ) are $17 \%$ higher than those of normal weight patients (95\% CI $0.3 \%$ to $34 \%$ ).

\section{DISCUSSION}

This study examines the annual healthcare costs in a wellcharacterised cohort of SRA patients and examines the distribution and drivers of costs in this population. A recent European study demonstrated mean per patient costs in controlled asthma of $€ 509$ ( $£ 451)$ and uncontrolled asthma $€ 2281$ ( $£ 2022)$, similar to our non-SRA cost. Patients with SRA cost more compared with other conditions (mean costs-type II diabetes $£ 2567$; stroke $£ 1301$; COPD $£ 819$; chronic kidney disease $£ 235$ ( $2 \%$ on renal replacement therapy $£ 27000$ )) (see online supplementary materials for full discussion and references).

A key finding of the study is that asthma medication is the major driver of total costs, not unscheduled GP/A\&E or hospital admissions. This implies that the overall cost of care in this population will significantly decrease if the price of multiple drug therapies used by them falls. This is likely to be most apparent for inhaled combination therapies, specifically with the arrival of generic combination inhalers. Finally, future studies examining the effect of novel therapies or interventions in SRA should consider a 'medication reduction' strategy in their development programme; specifically, if a new therapy could provide a reduction in healthcare utilisation in parallel with a reduction in maintenance asthma medication use, it is more likely to be cost-effective.

We found that subjects on maintenance OCS are $43 \%$ more expensive than those not receiving maintenance OCS. In those on maintenance steroids, asthma-related medications were more expensive but notably, their non-medication costs and non-asthma-related medication were significantly higher. Of note, non-asthma medication includes proton pump inhibitor and bisphosphonates, examples of therapies used to manage side effects of OCS induced morbidity. Recurrent exacerbation is also a significant driver of costs; subjects with more than two exacerbations requiring OCS were approximately 31\% more expensive than subjects with less than two courses of rescue OCS.

Establishing the costs and cost drivers for SRA will aid policymakers examining the cost-effectiveness of currently available 'add-on' treatments such as omalizumab in this group. Recent National Institute of Health and Care Excellence guidance on omalizumab allows access to subjects receiving recurrent rescue and maintenance OCS (see online supplementary materials for reference). Our data support this analysis, as both subjects requiring recurrent rescue OCS for exacerbation and maintenance OCS appear more expensive based on our results. However, it remains to be seen what additional costs are associated with longer term maintenance OCS in this population.

The association between severe obesity $(\mathrm{BMI}>40)$ and asthma cost is interesting - we have previously published data from the Registry that indicate patients with SRA and obesity display particular characteristics and may represent a distinct clinical phenotype. Cluster analyses of severe asthma populations have also identified obesity as an identifiable phenotype in severe asthma (see online supplementary materials for full discussion and references). The association with increased cost may reflect this altered phenotype and poor response to treatment.

Our analysis does have some limitations. Non-National Health Service and indirect costs were excluded, as were 
dispensing fees, and so our cost estimates are likely to be conservative. Similarly, as the Registry did not have complete records for all healthcare visits, assumptions around these were required; however, we do not think these threaten the validity of our analyses, as scheduled and unscheduled healthcare visits represent a minor element of total cost.

In conclusion, this paper has estimated treatment costs for SRA patients in the UK to be $£ 2912-£ 4217$ per person per year. These costs are greater than those for patients with poorly controlled 'difficult asthma' referred to the same clinics. The costs are dominated by asthma medication costs and, importantly, these costs outweigh healthcare utilisation costs. Maintenance OCS and frequent exacerbations are important additional drivers of costs in this population.

\section{Author affiliations}

${ }^{1} \mathrm{JE}$ Cairnes School of Business and Economics, National University of Ireland Galway, Galway, Ireland

${ }^{2}$ Centre for Infection and Immunity, Queen's University of Belfast, Belfast, UK

${ }^{3}$ Centre for Public Health, Queen's University of Belfast, Belfast, UK

${ }^{4}$ Royal Brompton Hospital, London, UK

${ }^{5}$ MAHSC, The University of Manchester \& UHSM, Manchester, UK

${ }^{6}$ Severe and Brittle Asthma Unit, Birmingham Heartlands Hospital, Birmingham, UK

${ }^{7}$ Department of Respiratory Medicine, Royal Infirmary, Glasgow, UK

${ }^{8}$ Department of Respiratory Medicine, Division of Immunology, Infection and Inflammation, University of Glasgow and Gartnavel General, Glasgow, UK

${ }^{9}$ Department of Infection, Inflammation and Immunity, Institute for Lung Health, University of Leicester, Leicester, UK

Acknowledgement Many thanks to the data input staff and medical and nursing staff in the UK Difficult Asthma Centres.

Contributors LGH is Co-ordinator of the British Thoracic Society UK Difficult Asthma Registry and with JS and SO'N collated and managed the data for this manuscript. LGH, JS, SO'N, $\mathrm{CO}^{\prime} \mathrm{N}$ and CCP were responsible for analysing the data. CEB, AM-G, RMN, AHM, CB, RC and NCT colead the British Thoracic Society Difficult Asthma; all have contributed equally to the writing and review of this manuscript.

Funding Pilot funding for the Registry was provided as unrestricted research grants from Astra Zeneca, GlaxoSmithKline, Novartis and Medimmune. JS is supported by HSC R\&D (NI) and GlaxoSmithKline (PhD studentship). CB is supported by a Wellcome Senior Clinical Fellowship.

Competing interests JS is supported by HSC R\&D (NI) and GlaxoSmithKline (PhD studentship funding). CEB is supported by a Wellcome Senior Clinical Fellowship and has received consultancy fees and or research funding from GlaxoSmithKline, $A Z$, Medlmmune, Amgen, Novartis, Chiesi, BI and Roche. AM-G has attended advisory boards for Mundi Pharma, Boeringer Ingelheim, Johnson and Johnson, Amgen and
Roche. He has received sponsorship to attend international meetings from Novartis and Boeringer Ingelheim. He has received lecture fees from Novartis,

GlaxoSmithKline, Roche and NAPP. He has taken part in asthma clinical trials (GSK and Genentech) for which his Institution was remunerated. CB has received travel support from Novartis, GlaxoSmithKline and Boehringer and has received payment for lectures, including service on speakers' bureaus, from Novartis and GlaxoSmithKline. RMN has received payment for lectures from GlaxoSmithKline, Novartis, Chiesi, Boehringer and AstraZeneca; and has received travel expenses from GlaxoSmithKline, Novartis and Boehringer. RMN (or any members of his family) has any shares or any percuniary interest in any pharmaceutical industry and has nothing to gain financially from the publication of this paper. AHM has received travel support from the British Thoracic Society; has consultant arrangements with NAPP and Novartis; has received payment for lectures, including service on speakers' bureaus, from GlaxoSmithKline, Chiesi, Vectura and Novartis; and has received travel expenses from Boehringer and Novartis. RC has received an unrestricted educational grant from Novartis to improve local asthma services, sponsorship to attend international meetings from GlaxoSmithKline, Novartis, Teva and Boeringer Ingelheim and has taken part in Advisory Board meeting (Novartis) and has taken part in asthma clinical trials (Roche, GSK, Astrazeneca, Janssen and Genentech) for which her Institution was remunerated. CCP's spouse holds shares in GlaxoSmithKline. NCT has participated in advisory boards and/or received consultancy fees from Chiesi, GlaxoSmithKline, Respivert and Roche. He has received lecture fees from AstraZeneca, Boston Scientific, Chiesi, Glaxo SmithKline and Novartis; industry-sponsored grant funding to the University of Glasgow from AstraZeneca, Boston Scientific, Genentech, Glaxo SmithKline, Novartis, Pfizer, Respivert and Synairgen for participating in clinical trials. Professor Heaney has received grant funding from Genentech and GlaxoSmithKline, has taken part in Advisory Boards and given lectures at meetings supported by GlaxoSmithKline, Merck Sharpe \& Dohme, Nycomed, Novartis and Astra Zeneca. He has received support funding to attend International Respiratory meetings (AstraZeneca, Chiesi, Novartis, Teva and GlaxoSmithKline) and has taken part in asthma clinical trials (GSK and Genentech) for which his Institution was remunerated. None of these activities have any direct relationship to the content of this manuscript.

Ethics approval ORECNI.

Provenance and peer review Not commissioned; externally peer reviewed.

\section{REFERENCES}

1 Global Initiative for Asthma. Global strategy for asthma management and prevention. 2012. http://www.ginasthma.org/local/uploads/files/GINA_Report_ March13.pdf (accessed 31 Jul 2013).

2 To T, Stanojevic S, Moores G, et al. Global asthma prevalence in adults: findings from the cross-sectional world health survey. BMC Public Health 2012;12:204.

3 Bahadori K, Doyle-Waters MM, Marra C, et al. Economic burden of asthma: a systematic review. BMC pulmonary medicine 2009;9:24.

4 Cisternas MG, Blanc PD, Yen $\mathrm{IH}$, et al. A comprehensive study of the direct and indirect costs of adult asthma. J Allergy Clin Immunol 2003;111:1212-18.

5 American Thoracic Society. Proceedings of the ATS workshop on refractory asthma: current understanding, recommendations, and unanswered questions. Am J Respir Crit Care Med 2000;162:2341-51. 